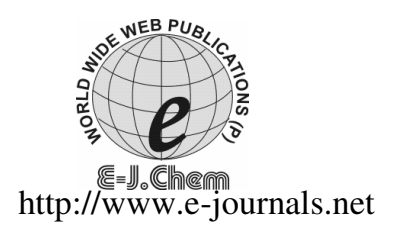

ISSN: 0973-4945; CODEN ECJHAO

E-Journal of Chemistry

2011, 8(2), 863-869

\title{
Facile Condensation of Aromatic Aldehydes with Chroman-4-ones and 1-Thiochroman-4-ones Catalysed by Amberlyst-15 under Microwave Irradiation Condition
}

\author{
TAPAS K. MANDAL, RAMMOHAN PAL, \\ RINA MONDAL and ASOK K. MALLIK* \\ Department of Chemistry \\ Jadavpur University, Kolkata-700032, India \\ mallikak52@yahoo.co.in
}

Received 17 August 2010; Accepted 8 November 2010

\begin{abstract}
Different aromatic aldehydes and cinnamaldehyde undergo crossaldol condensation with chroman-4-ones and 1-thiochroman-4-ones in the presence of amberlyst- 15 under microwave irradiation in solvent free condition to afford rapidly the corresponding E-3-arylidene and E-3-cinnamylidene derivatives, respectively, in high yield. This process is simple, efficient and environmentally benign.
\end{abstract}

Keywords: Amberlyst-15, Microwave irradiation, E-3-Arylidenechroman-4-ones, E-3-Cinnamylidene chroman-4-ones, E-3-Arylidenethiochroman-4-ones, E-3-Cinnamylidenethiochroman-4-ones.

\section{Introduction}

Both natural and synthetic chromone derivatives are known to show important biological activities $^{1,2}$. Thiochromones are mostly synthetic compounds and some of their derivatives are reported to show medicinal properties ${ }^{3,4}$. Current literature shows that there has been a growing trend towards synthesis of heterocycles containing these two ring systems ${ }^{5}$. The corresponding reduced systems, chroman-4-ones and 1-thiochroman-4-ones possess a ketomethylene moiety. Condensation of aldehydes at this moiety appears to be an entry point for synthesis of complex molecules containing chromone and thiochromone units. In this connection special mention may be made for the synthesis of natural homoiso flavonoids, some of which are known to act as anti-inflammatory and cytotoxic agents ${ }^{6}$. The said condensation has been reported to be performed in a number of ways. The initial methods were base-catalysed processes ${ }^{7}$ and subsequently, acid-catalysed methods have also been developed ${ }^{8}$. Modifications like condensation using acetic anhydride ${ }^{9}$ or piperidine ${ }^{10}$ 
have also been reported. The acid-catalysed processes are sometimes two-step processes, the first step giving an aldol which is required to be dehydrated subsequently ${ }^{11}$. Moreover, isomerisation $^{12}$ and LAH reduction ${ }^{11,13}$ of these $E$-3-arylidene derivatives have been studied with much interest. Amberlyst-15 is a sulphonated polystyrene resin ${ }^{14}$ that has been used for a large number of transformations promoted by strong acids. Microwave irradiation is an efficient and environmentally benign method to activate various organic transformations to afford products in higher yields in shorter reaction periods. Among the MW-assisted reactions, solvent free processes are of particular interest and importance in view of their simplicity, tunability and ease of work-up ${ }^{15}$. Herein we report a new method for synthesis of $E$-3-arylidenechroman-4-ones and E-3-arylidene-1-thiochroman-4-ones with different aromatic aldehydes and cinnamaldehyde done by use of amberlyst-15 as catalyst under microwave irradiation in solvent free condition (Schemes $1 \& 2$ ).<smiles>[R]c1ccc([X])c(C(=O)CCC)c1</smiles>

1<smiles>[R]c1ccc2c(c1)C(=O)CC[X]2</smiles>

1

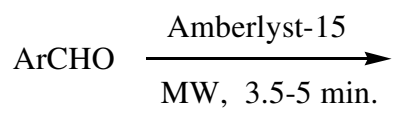

Scheme 1

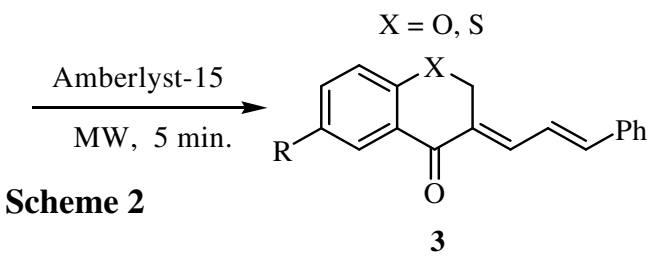

\section{Experimental}

$\mathrm{X}=\mathrm{O}, \mathrm{S}$

All melting points were recorded on a Köfler block and are uncorrected. IR spectra were recorded on Perkin-Elmer FT-IR spectrophotometer (Spectrum RX 1) in $\mathrm{KBr}$ pellets. ${ }^{1} \mathrm{H}$ and ${ }^{13} \mathrm{C}$ NMR spectra were recorded in $\mathrm{CDCl}_{3}$ on a Bruker AV-300 (300 MHz) spectrometer using TMS as internal standard. Analytical samples were routinely dried in vacuo at room temperature. Column chromatography was performed with silica gel (100-200 mesh) and TLC with silica gel G made of SRL Pvt. Ltd. Petroleum ether had the boiling range $60-80{ }^{\circ} \mathrm{C}$. Amberlyst-15 used was made of fluka chemika. An unmodified domestic household microwave oven (LG, DMO, Model No.-556P, 900 watt) equipped with inverter technology, which provides a realistic control of the microwave power to the desired level $(20 \%-100 \%)$ was used for microwave heating. The MW oven was operated at reduced MW-power level of $60 \%$ (540 watt) and total power level $100 \%$ (900 watt).

\section{General procedure for condensation reactions}

In a typical procedure, a mixture of chroman-4-one / 1-thiochroman-4-one (2 mmol), aromatic aldehyde / cinnamaldehyde ( $2 \mathrm{mmol})$, neutral alumina (5 g) and Amberlyst-15 (100 mg) were thoroughly mixed and the whole mixture was taken in a Pyrex beaker $(20 \mathrm{~mL})$. The mixture was then irradiated in the microwave oven for appropriate time. After irradiation the mixture was cooled, shaken with chloroform $(10 \mathrm{~mL})$ and filtered. The filtrate was concentrated and subjected to rapid column chromatography over silica gel using petroleum ether-ethyl acetate (9:1) as eluent to obtain the crystalline pure product. Majority of the products were known compounds and some of them were not known previously. The products were characterized from their literature melting points, physical, analytical and spectral (IR and ${ }^{1} \mathrm{H}$ and ${ }^{13} \mathrm{C} N M R$ ) data. The spectral data of some of the compounds are given below: 
Compound 2f : Yellow crystals; IR (KBr) $\mathrm{cm}^{-1}$ : $1666(\mathrm{C}=\mathrm{O}),{ }^{1} \mathrm{H}$ NMR $(300 \mathrm{MHz}$, $\left.\mathrm{CDCl}_{3}\right): \delta 5.34\left(2 \mathrm{H}, \mathrm{s}, \mathrm{H}_{2}-2\right), 6.02\left(\mathrm{OCH}_{2} \mathrm{O}\right), 6.78(1 \mathrm{H}$, br. s, H-2'), $6.82(1 \mathrm{H}, \mathrm{d}, J=8.2 \mathrm{~Hz}$, H-5'), $6.88\left(1 \mathrm{H}, \mathrm{d}, J=8 \mathrm{~Hz}, \mathrm{H}-6^{\prime}\right), 6.95(1 \mathrm{H}, \mathrm{d}, J=8.3 \mathrm{~Hz}, \mathrm{H}-8), 7.06(1 \mathrm{H}$, br. t, $J=7.5 \mathrm{~Hz}$, $\mathrm{H}-6), 7.47(1 \mathrm{H}$, br. t, $J=7.7 \mathrm{~Hz}, \mathrm{H}-7), 7.77(1 \mathrm{H}$, br. s, H- $\beta)$ and $8.00(1 \mathrm{H}, \mathrm{d}, J=7.8 \mathrm{~Hz}$, $\mathrm{H}-5) .{ }^{13} \mathrm{C}$ NMR $\left(75 \mathrm{MHz}, \mathrm{CDCl}_{3}\right) \delta 67.7,101.6,108.6,109.8,117.8,121.8,122.1,125.4$, 127.9, 128.5, 129.4, 135.7, 137.3, 148.1, 148.9, 160.9 and $182.0(\mathrm{C}=\mathrm{O})$. Anal. (\%) Calcd. for $\mathrm{C}_{17} \mathrm{H}_{12} \mathrm{O}_{4}$ (280.27): C, 72.85: H, 4.32. Found C, 72.56: H, 4.44.

Compound 2l : Yellow crystals; IR (KBr) $\mathrm{cm}^{-1}: 1660(\mathrm{C}=\mathrm{O}),{ }^{1} \mathrm{H}$ NMR $(300 \mathrm{MHz}$, $\left.\mathrm{CDCl}_{3}\right): \delta 2.33\left(3 \mathrm{H}, \mathrm{s}, \mathrm{CH}_{3}\right), 3.05\left(6 \mathrm{H}, \mathrm{s}, \mathrm{NMe}_{2}\right), 5.39\left(2 \mathrm{H}, \mathrm{s}, \mathrm{H}_{2}-2\right), 6.55(1 \mathrm{H}$, br. s, H-3'), $6.72\left(2 \mathrm{H}, \mathrm{d}, J=8.7 \mathrm{~Hz}, \mathrm{H}-3^{\prime} \& \mathrm{H}-5^{\prime}\right), 6.85(1 \mathrm{H}, \mathrm{d}, J=8.3 \mathrm{~Hz}, \mathrm{H}-7), 7.25-7.27\left(3 \mathrm{H}, \mathrm{H}-8, \mathrm{H}-2^{\prime}\right.$ \& H-6'), 7.81(1H, s, H- $\beta$ ), 7.82(1H, s, H-5) Anal.(\%) Calcd. for $\mathrm{C}_{19} \mathrm{H}_{19} \mathrm{O}_{2} \mathrm{~N}$ (293.36): $\mathrm{C}$, 77.79: H, 6.53, N, 4.77. Found C, 77.60: H, 6.45: N, 4.68.

Compound 2m : Yellow crystals; IR $(\mathrm{KBr}) \mathrm{cm}^{-1}: 1670(\mathrm{C}=\mathrm{O}),{ }^{1} \mathrm{H}$ NMR $(300 \mathrm{MHz}$, $\left.\mathrm{CDCl}_{3}\right): \delta 2.31\left(3 \mathrm{H}, \mathrm{s}, \mathrm{CH}_{3}\right), 5.31\left(2 \mathrm{H}, \mathrm{s}, \mathrm{H}_{2}-2\right), 6.03\left(\mathrm{OCH}_{2} \mathrm{O}\right), 6.79-7.30(\mathrm{Ar}-\mathrm{H}), 7.77(1 \mathrm{H}$, br. s, H- $\beta$ ) and 7.79 (1H, s, H-5). Anal. (\%) Calcd. for $\mathrm{C}_{18} \mathrm{H}_{14} \mathrm{O}_{4}$ (294.30): C, 73.46: H, 4.79. Found C, 73.36: H, 4.86.

Compound 2n : Yellow needles; IR (KBr) $\mathrm{cm}^{-1} 1667(\mathrm{C}=\mathrm{O}),{ }^{1} \mathrm{H}$ NMR (300 MHz, $\left.\mathrm{CDCl}_{3}\right): \delta 2.33\left(3 \mathrm{H}, \mathrm{s}, \mathrm{CH}_{3}\right), 5.67\left(2 \mathrm{H}, \mathrm{s}, \mathrm{H}_{2}-2\right), 6.55\left(1 \mathrm{H}\right.$, br. s, $\left.\mathrm{H}-4^{\prime}\right), 6.74(1 \mathrm{H}, \mathrm{d}, J=3.1$ $\left.\mathrm{Hz}, \mathrm{H}-3^{\prime}\right), 6.89(1 \mathrm{H}, \mathrm{d}, J=8.4 \mathrm{~Hz}, \mathrm{H}-8), 7.28(1 \mathrm{H}$, br. d, $J=8.4 \mathrm{~Hz}, \mathrm{H}-7$ ), 7.51, 7.61 and 7.79 (each $1 \mathrm{H}$, br. s, H-5, H- $\beta$ and $\mathrm{H}^{-5}$ ). Anal. (\%) Calcd. for $\mathrm{C}_{15} \mathrm{H}_{12} \mathrm{O}_{3}$ (240.25): C, 74.99: H, 5.03. Found C, 74.76: H, 4.92.

Compound 3c : Yellow crystals; IR $(\mathrm{KBr}) \mathrm{cm}^{-1}: 1664(\mathrm{C}=\mathrm{O}),{ }^{1} \mathrm{H}$ NMR $(300 \mathrm{MHz}$, $\left.\mathrm{CDCl}_{3}\right): \delta 2.33\left(3 \mathrm{H}, \mathrm{s}, \mathrm{CH}_{3}\right), 5.23\left(2 \mathrm{H}, \mathrm{s}, \mathrm{H}_{2}-2\right), 6.86-7.10(3 \mathrm{H}, \mathrm{m}, \mathrm{H}-8, \mathrm{H}-\gamma$ \& H- $\delta$ ), 7.277.40 (4H, m, Ar-H), 7.47-7.56 (3H, m, H- $\beta$ \& Ar-H), 7.80 (1H, br. s, H-5). Anal.(\%) Calcd. for $\mathrm{C}_{19} \mathrm{H}_{16} \mathrm{O}_{2}$ (276.33): C, 82.58: H, 5.84. Found C, 82.45: H, 5.98.

\section{Results and Discussion}

A number of aromatic aldehydes as well as cinnamaldehyde underwent rapid cross-aldol condensation with 4-chromanones and 1-thio-4-chromanone when subjected to microwave irradiation in the presence of amberlyst- 15 on neutral alumina. The products were $E$-3-arylidene and E-3-cinnamylidene derivatives of chroman-4-ones and 1-thiochroman-4-ones and their yields were very good to excellent (Tables $1 \& 2$ ). The reactions were performed in solvent free condition. They were very fast (complete within 3.5-5 minutes), clean and environmentally benign. Conversions were partial when these reactions were carried out under thermal condition for $4 \mathrm{~h}$. This clearly indicated the role of microwave in enhancing the reaction rates as well as the yields. Thus, microwave irradiation, which has become a powerful synthetic tool for the preparation of various biologically active molecules under solvent-free conditions, can also be used for the preparation of many classical organic reactions. Among various materials providing solid surface, neutral alumina is attractive because of its low cost and nontoxic nature. Moreover, it is very simple to apply during reactions and can be reused.

In a previous method for synthesis of compounds $\mathbf{2}$, their isomerisation to corresponding endocyclic compounds [i.e., to 3-(arylmethyl)chromones or 3-(arylmethyl)thiochromones] were reported ${ }^{18}$. In the present method no trace of such isomerisation was observed (3-(arylmethyl)chromones and 3-(arylmethyl)thiochromones are known to give a two-proton singlet around $\delta 3.85$ and 4.00, respectively, for their methylene protons ${ }^{18}$. However, any product giving such spectral features could not be isolated by us). 
Table 1. Microwave assisted synthesis of E-3-arylidenechroman-4-ones and E-3-arylidene1-thiochroman-4-ones

\begin{tabular}{|c|c|c|c|c|c|c|}
\hline Entry $^{\dagger}$ & $\begin{array}{l}\text { Ketone } \\
\text { (1) }\end{array}$ & Aldehyde & $\begin{array}{l}\text { Time } \\
\text { min. }\end{array}$ & $\begin{array}{l}\text { Product } \\
\text { (2) }\end{array}$ & $\begin{array}{c}\text { Yield, } \\
\%\end{array}$ & $\begin{array}{c}\text { M.P. }{ }^{\circ} \mathrm{C} \\
\text { Obs. (Lit.) }\end{array}$ \\
\hline $\mathbf{a}$ & Chr. & & 4.0 & & 89 & $\begin{array}{c}111 \\
(110)^{16}\end{array}$ \\
\hline b & Chr. & & 3.5 & & 91 & $\begin{array}{c}170 \\
(168)^{16}\end{array}$ \\
\hline c & Chr. & & 5.0 & & 85 & $\begin{array}{c}131 \\
(132)^{16}\end{array}$ \\
\hline d & Chr. & & 4.0 & & 88 & $\begin{array}{c}175 \\
(174)^{16}\end{array}$ \\
\hline $\mathbf{e}$ & Chr. & & 5.0 & & 85 & $\begin{array}{c}116 \\
(118)^{16}\end{array}$ \\
\hline f & Chr. & & 4.5 & & 84 & 115 \\
\hline g & Chr. & & 5.0 & & 81 & 105 \\
\hline h & Chr. & & 3.5 & & 92 & $\begin{array}{c}223 \\
(223)^{16}\end{array}$ \\
\hline $\mathbf{i}$ & $\begin{array}{c}\text { 6-Me- } \\
\text { Chr. }\end{array}$ & & 5.0 & & 88 & 133 \\
\hline $\mathbf{j}$ & $\begin{array}{l}\text { 6-Me- } \\
\text { Chr. }\end{array}$ & & 3.5 & & 91 & 144 \\
\hline $\mathbf{k}$ & $\begin{array}{c}\text { 6-Me- } \\
\mathrm{Chr}\end{array}$ & & 4.5 & & 86 & 120 \\
\hline 1 & $\begin{array}{l}\text { 6-Me- } \\
\text { Chr. }\end{array}$ & & 5.0 & & 90 & 140 \\
\hline $\mathbf{m}$ & $\begin{array}{l}\text { 6-Me- } \\
\text { Chr. }\end{array}$ & & 4.5 & & 82 & $\begin{array}{c}135 \\
*\end{array}$ \\
\hline n & $\begin{array}{l}\text { 6-Me- } \\
\text { Chr. }\end{array}$ & & 5.0 & & 78 & $\begin{array}{c}101 \\
*\end{array}$ \\
\hline $\mathbf{o}$ & $\begin{array}{l}\text { 6-Cl- } \\
\text { Chr. }\end{array}$ & & 4.0 & & 87 & $\begin{array}{c}134 \\
*\end{array}$ \\
\hline
\end{tabular}




(2)

${ }^{\dagger}$ Chr. = Chroman-4-one, 6-Me-Chr. = 6-Methylchroman-4-one, 6-Cl-Chr. = 6-Chlorochroman-4-one, Th-chr. $=1$-Thiochroman-4-one $*$ Showed the following characteristic ${ }^{1} H$ NMR signals: $2 H$, s, around $\delta 5.35\left(H_{2}-2\right) ; 1 H$, $s$, around $\delta 7.80(H-\beta)$

Table 2. Microwave assisted synthesis of E-3-cinnamylidenechroman-4-ones and E-3cinnamylidene-1-thiochroman-4-ones

\begin{tabular}{ccccccc}
\hline Entry $^{\dagger}$ & $\begin{array}{c}\text { Ketone } \\
(\mathbf{1})\end{array}$ & Aldehyde & $\begin{array}{c}\text { Time, } \\
\text { min. }\end{array}$ & $\begin{array}{c}\text { Product } \\
(\mathbf{3})\end{array}$ & $\begin{array}{c}\text { Yield } \\
\%\end{array}$ & $\begin{array}{c}\text { M.P, }{ }^{\circ} \mathrm{C} \\
\text { Obs. (Lit.) }\end{array}$ \\
\hline b & Chr. & $\begin{array}{c}\text { Cinnamal- } \\
\text { dehyde }\end{array}$ & 5.0 & $\begin{array}{c}\text { Cinnamal- } \\
\text { dehyde }\end{array}$ & 5.0 &
\end{tabular}

${ }^{\dagger}$ Chr. $=$ Chroman-4-one, 6-Me-Chr. =6-Methylchroman-4-one, 6-Cl-Chr. $=6$ - Chlorochroman-4-one, Th-chr. = 1-Thiochroman-4-one 


\section{Conclusion}

A new methodology for the synthesis of E-3-arylidene and $E$-3-cinnamylidene derivatives of chroman-4-ones and 1-thiochroman-4-ones has been developed. The method is very efficient, simple and environmentally benign.

\section{Acknowledgment}

Financial assistance from the CAS programme, Department of Chemistry is gratefully acknowledged. The authors also acknowledge the DST-FIST programme to the Department of Chemistry, Jadavpur University for providing the NMR spectral data.

\section{References}

1. (a) McClure J W, Harborne J B, Mabry T J and Mabry H (Eds.), The Flavonoids, Chapman and Hall: London, 1975, 970-1055; (b) Atassi G, Briet P, Berthelon J P and Collonges F, J Med Chem.,1985, 20, 393; (c) Middleton Jr. E, Kandaswami C and Harborne J B (Eds.), The Flavonoids Advances in Research Since 1986, Chapman and Hall: London, 1994, 619; (d) Bruneton J, Pharmacognosy, Phytochemistry and Medicinal Plants, English Translation by Hatton C K, Lavoisier Publishing, Paris, 1995, 265; (e) Harborne J B and Williams C A, Phytochem., 2000, 55(6), 481-504.

2. (a) Gupta A, Dwivedy A, Keshri G and Sharma R, Balapure A K, Singh M M and Ray S, Bioorg Med Chem Lett., 2006, 16, 6006-6012; (b) Foroumadi A, SamzadehKermani A, Emami S, Dehghan G, Sorkhi M, Arabsorkhi F, Heidari M R, Abdollahi M and Shafiee A, Bioorg Med Chem Lett., 2007, 17, 6764-6769.

3. (a) Katrizky A R and Boulton A J, J Adv Heterocycl Chem., 1975, 18, 76; (b) Buston G W, Doba T, Gabe E J, Huges L, Lee F, Prasad L and Ingold K U, J Am Chem Soc., 1985, 107, 7053.

4. (a) Wang H-K, Bastow K F, Cosentino L M and Lee K-H, J Med Chem., 1996, 39, 1975-1980; (b) Liu Y, Luo W, Sun L and Guo C, Drug Discov Ther., 2008, 2(4), 216-218.

5. (a) Ghosh C K and Patra A, J Heterocycl Chem., 2008, 45, 1529-1547, (b) Ibrahim M A, Ali T E, Alnamer Y A and Gaber Y A, Arkivoc, 2010(xi), 98, (c) Li N-G, Shi Z-H, Tang Y-P, Ma H-Y, Yang J-P, Li B-Q, Wang Z-J, Song S-L and Duan J-A, $J$ Heterocycl Chem., 2010, 47, 785, (d) Wang W, Li H, Wang J and Zu L, J Am Chem Soc., 2006, 128, 10354-10355.

6. Yen C-T, Nakagawa-Goto K, Hwang T-L, Wa P-C, Morris-Natschke S L, Lai W-C, Bastow K F, Chang F-R, Wu Y-C and Lee K-H, Bioorg Med Chem Lett., 2010, 20(3), 1037-1039.

7. (a) Pfeiffer P and Emmer H J, Chem Ber., 1920, 53B, 945; (b) Pfeiffer P, Oberlin H and Konermann E, Chem Ber., 1925, 58B, 1947.

8. (a) Arndt F and Kallner G, Chem Ber., 1924, 57B, 202; (b) Dann O and Hofmann H, Chem Ber. 1962, 95, 1446; (c) Dann O and Hofmann H, Chem Ber., 1965, 98, 1498, (d) Evans D and Lockhart I M, J Chem Soc., 1966, 711; (e) Pfeiffer P, Breitb E and Hoyer H, J Prakt Chem., 1931, 31, 129; (f) Pfeiffer P and Dobring E, Chem Ber., 1938, 71, 279; (g) Pfeiffer P, Grimm K and Schmidt H, Liebigs Ann Chem., 1949, 564, 208; (h) Wagner G, Garbe C, Vieweg H, Brunn M and Dittrich A, Pharmazie, 1979, 34, 55; (i) Wagner G, Horn B, Eppner H and Kuhmstedt H, Pharmazie, 1979, 34, 56.

9. (a) Farkas L, Gottsegen A and Nogradi M, Tetrahedron Lett., 1968, 4099; (b) Farkas L, Gottsegen A and Nogradi M, Tetrahedron., 1970, 26, 2787, (c) Farkas L, Gottsegen A, Nogradi M and Strelisky J, Tetrahedron., 1971, 27, 5049. 
10. (a) Levai A and Hetey E H, Pharmazie, 1978, 33, 378; (b) Levai A and Schag J B, Pharmazie, 1979, 34, 749; (c) Levai A, Szollosy A and Toth G, Acta Chim Hung., 1991, 128, 359-364, (d) Levai A, Arkivoc, 2004(vii), 15.

11. Chatterjee A, Dutta L N and Chatterjee S K, Indian J Chem., 1980, 19B, 955-960.

12. (a) Mulragh D, Meegan M J and Donnely D, J Chem Res M, 1979, 1713-1731; (b) Jain A C, Shrma A and Srivastava R, Indian J Chem., 1983, 22B, 1119.

13. (a) Gomis M, Kirkiacharian B S, Likforman J and Mahuteau J, Bull Soc Chim., France, 1988, 585-590; (b) Koch K and Smiprovich J H, Tetrahedron Lett., 1994, 35, 1137-1140.

14. Amberlyst-15 Synthetic Resin Catalyst, Technical Bulletin-Fluid Process Chemicals, Rohm and Haas Company, Inc., Philadelphia, PA, 1978.

15. (a) Caddick S, Tetrahedron, 1995, 51, 10403; (b) Strauss C. R and Trainor R. W, Aust J Chem., 1995, 48, 1665; (c) Galema S A, Chem Soc Rev., 1997, 26, 233-238; (d) Loupy A, Petit A, Hamelin J, Texier-Boullet F, Jacqualt P and Mathe D, Synthesis, 1998, 1213; (e) Varma R S, Green Chem., 1999, 43-55; (f) Varma R S, Pure Appl Chem., 2001,73, 193-198; (g) Varma R S, Tetrahedron, 2002, 58, 1235.

16. Hamman G A E-F, Fahmy A F M, Amr A-G E and Mohamad A M, Indian J Chem., 2003, 42(8)B, 1985-19893.

17. Levai A, Simon A, Jenei A, Kalmen G, Jeko J and Toth G, Arkivoc, 2009(xii), 161.

18. Levai A, Dinya Z, Schag J B, Toth G and Szollosy A, Pharmazie, 1981, 36, 465. 


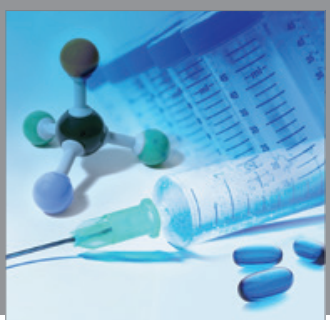

International Journal of

Medicinal Chemistry

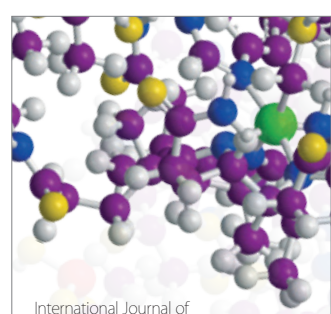

Carbohydrate Chemistry

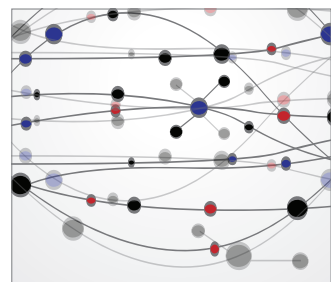

The Scientific World Journal
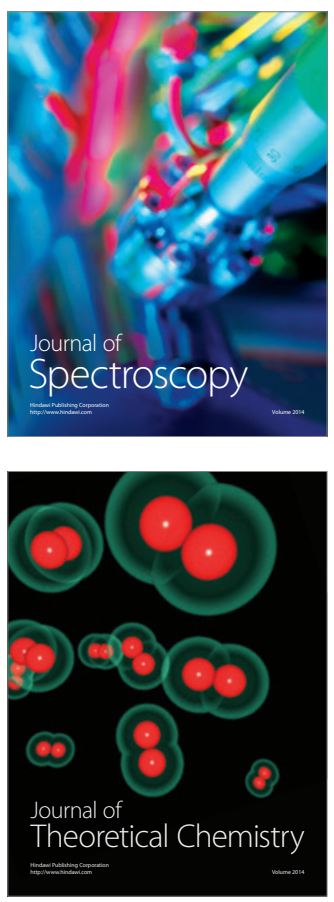
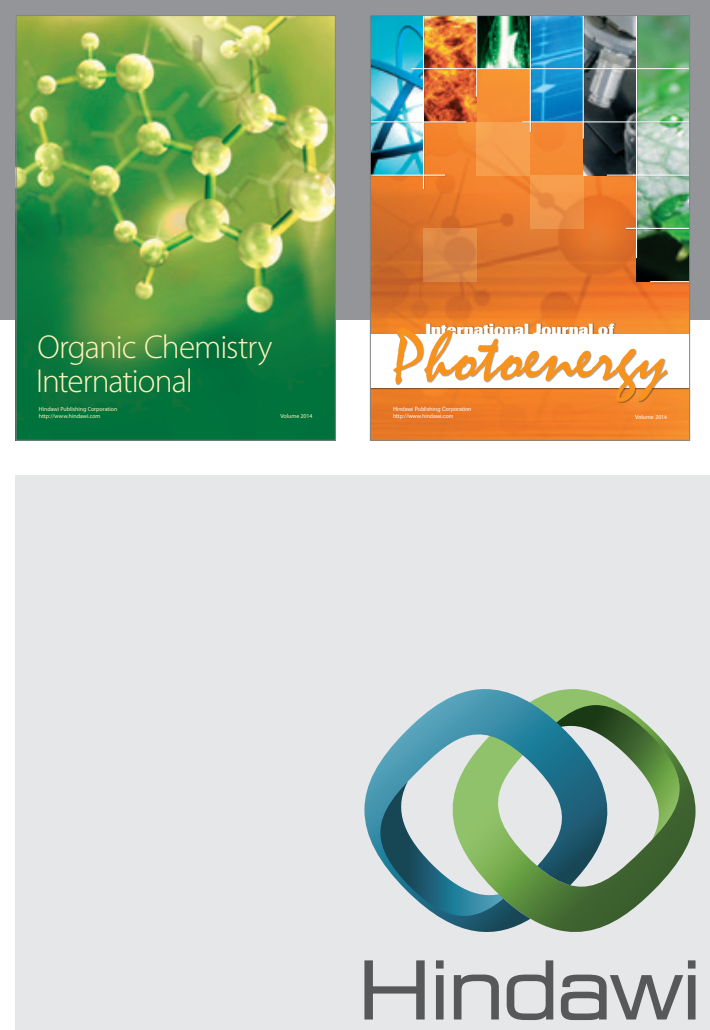

Submit your manuscripts at

http://www.hindawi.com
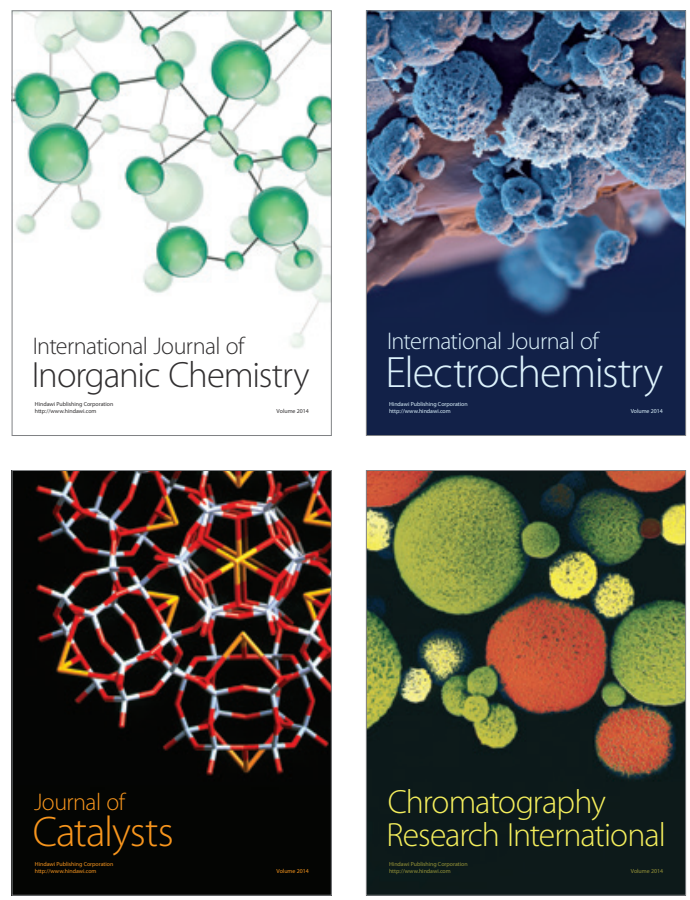
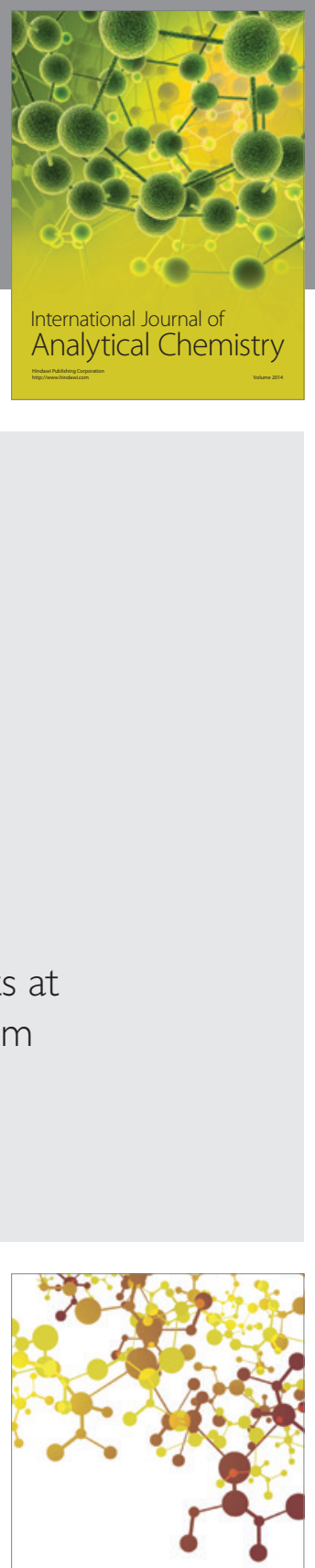

Journal of

Applied Chemistry
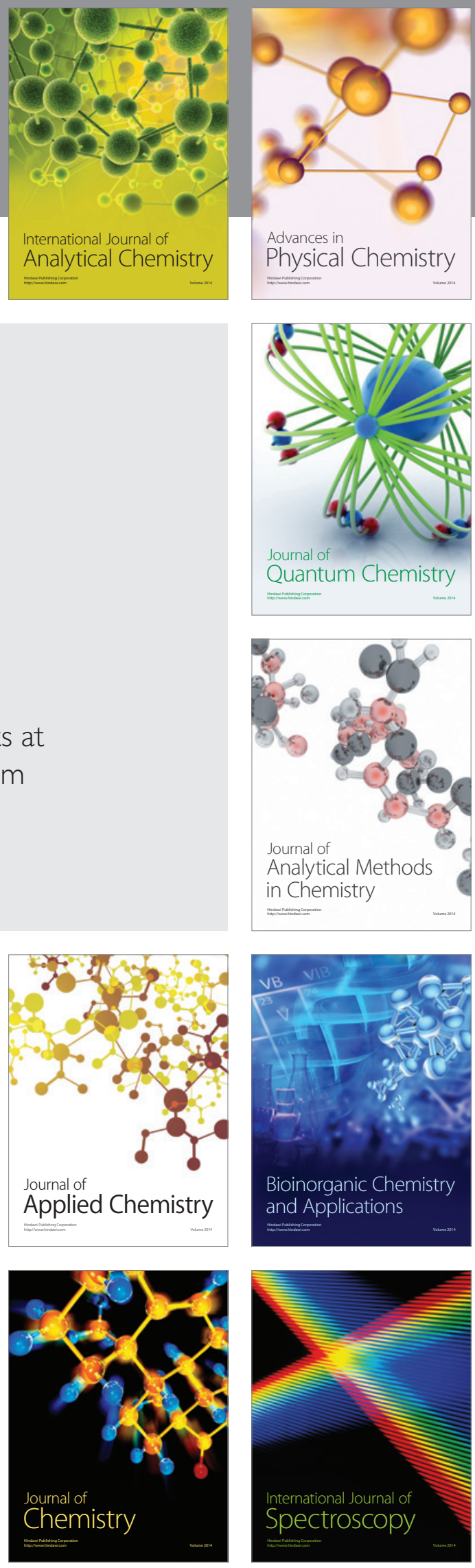